\title{
Reductions in tyrosine levels are associated with thyroid hormone and catecholamine disturbances in sepsis
}

\author{
W Khaliq ${ }^{1 *}$, DT Andreis ${ }^{1,2}$, A Kleyman $^{3}, M$ Gräler $^{3}$, M Singer $^{1}$ \\ From ESICM LIVES 2015 \\ Berlin, Germany. 3-7 October 2015
}

\section{Introduction}

The magnitude of fall in thyroid hormone and rise in catecholamine levels are prognostic in critical illness $(1,2)$. These may be linked, as the amino acid tyrosine is converted by tyrosine hydroxylase to L-DOPA, and thence to dopamine, norepinephrine and epinephrine, whereas thyroxine (T4) and tri-iodothyronine (T3) are formed by the iodination of tyrosine residues in thyroglobulin. We used our well-characterized $72 \mathrm{~h}$ fluid-resuscitated rat model of faecal peritonitis, where accurate prognostication can be made as early as $6 \mathrm{~h}(3)$, to explore any relationship.

\section{Objectives}

To determine if any association exists between early changes in circulating tyrosine, thyroid hormone and catecholamine levels, and eventual outcome in this rat peritonitis model.

\section{Methods}

Awake, instrumented yet fully mobile male Wistar rats $(325 \pm 15 \mathrm{~g})$ received an i.p. injection of $4 \mu \mathrm{l} / \mathrm{g}$ faecal slurry.
Fluid resuscitation (50:50 mix of 5\% glucose/Hartmann's; $10 \mathrm{ml} / \mathrm{kg} / \mathrm{h}$ ) was commenced at $2 \mathrm{~h}$. At $6 \mathrm{~h}$, an echomeasured heart rate cut-off of $460 \mathrm{bpm}$ was used to classify animals into predicted survivors or non-survivors. Blood samples were also taken for measurement of plasma L-tyrosine (mass spectrometry), thyroid hormone and catecholamine levels (ELISA). Control animals were treated identically except for slurry injection. Results were analysed using two-way ANOVA and post-hoc testing and considered statistically significant when $\mathrm{p}<0.05$.

\section{Results}

Predicted survivor and non-survivor septic animals were clinically indistinguishable at $6 \mathrm{~h}$. Tyrosine levels were similarly low and catecholamine levels similarly elevated in both septic subgroups $(\mathrm{p}<0.05)$, whereas non-survivors had significantly lower levels of T3 and T4 ( $<0.05)$.

\section{Conclusions}

Sepsis resulted in significant early reductions in circulating tyrosine levels. The elevation of plasma catecholamines

Table 1

\begin{tabular}{|c|c|c|c|}
\hline & Control $(n=6)$ & Predicted survival $(n=6)$ & Predicted non-survival $(n=6)$ \\
\hline L-Tyrosine $(\mu \mathrm{mol} / \mathrm{L})$ & $90.2 \pm 3.3$ & $58.8 \pm 3.3^{a}$ & $62.7 \pm 1.3^{a}$ \\
\hline $\mathrm{T} 3(\mathrm{pg} / \mathrm{mL})$ & $3.20 \pm 0.41$ & $2.77 \pm 0.44$ & $1.27 \pm 0.25^{a, b}$ \\
\hline T4 (ng/mL) & $9.20 \pm 0.75$ & $7.19 \pm 0.50^{a}$ & $5.03 \pm 1.02^{a, b}$ \\
\hline TSH (ng/mL) & $1.25 \pm 0.30$ & $1.55 \pm 0.21$ & $1.64 \pm 0.15$ \\
\hline Adrenaline $(\mathrm{ng} / \mathrm{mL})$ & $8.56 \pm 0.42$ & $9.44 \pm 0.23$ & $10.3 \pm 0.18$ \\
\hline Noradrenaline $(\mathrm{ng} / \mathrm{mL})$ & $1.60 \pm 0.25$ & $3.21 \pm 0.23^{a}$ & $2.98 \pm 0.27^{a}$ \\
\hline \multicolumn{4}{|c|}{ Data shown as median $\pm S E ;{ }^{a} p<0.05$ versus control, ${ }^{b} p<0.05$ versus survivors } \\
\hline
\end{tabular}

${ }^{1}$ University College London, Bloomsbury Institute of Intensive Care Medicine, London, United Kingdom 
suggests increased hydroxylation of tyrosine, while reductions in thyroid hormone levels may be due to reduced tyrosine iodination. This relationship has not, to our knowledge, been previously described in sepsis and warrants further investigation.

\section{Grant Acknowledgment}

ESICM Basic Science Award, Intensive Care Foundation (UK), NIHR.

\section{Authors' details}

${ }^{1}$ University College London, Bloomsbury Institute of Intensive Care Medicine, London, United Kingdom. ${ }^{2}$ Università degli Studi di Milano, Dipartimento di Fisiopatologia Medico-Chirurgica e dei Trapianti, Milan, Italy. ${ }^{3}$ University Hospital Jena, SG Sepsis Research, Department of Anesthesiology and Intensive Care Medicine, Jena, Germany.

Published: 1 October 2015

\section{References}

1. Bacci V, et al: J Clin Endocrinol Metab 1982, 54:1229-35.

2. Hahn P, et al: Shock 1995, 4:269-73.

3. Rudiger A, et al: Clin Sci 2013, 124:391-401.

doi:10.1186/2197-425X-3-S1-A686

Cite this article as: Khaliq et al:: Reductions in tyrosine levels are associated with thyroid hormone and catecholamine disturbances in sepsis. Intensive Care Medicine Experimental 2015 3(Suppl 1):A686.

\section{Submit your manuscript to a SpringerOpen ${ }^{\mathcal{O}}$ journal and benefit from:}

- Convenient online submission

- Rigorous peer review

- Immediate publication on acceptance

- Open access: articles freely available online

- High visibility within the field

- Retaining the copyright to your article 